\title{
Conceptualizaciones de la Pendiente en Libros de Texto de Matemáticas
}

\author{
Slope Conceptualizations in Mathematics Textbooks
}

\author{
Crisólogo Dolores Flores* \\ ORCID iD 0000-0002-2748-6042 \\ Gerardo Ibáñez Dolores ** \\ ORCID iD 0000-0002-3074-1008
}

\begin{abstract}
Resumen
En este artículo se reportan los resultados de una investigación que tiene por objetivo explorar qué conceptualizaciones de pendiente tienen presencia en los libros de texto de matemáticas del bachillerato y cuáles predominan. Para ello utilizamos el método de Análisis de Contenido, en donde los objetos de análisis se encuentran en la exposición del contenido, ejemplos resueltos y los ejercicios o problemas propuestos en los libros de texto. Como marco referencial utilizamos las once conceptualizaciones de pendiente identificadas por Stump (1999) y Moore-Russo, Conner y Rugg (2011). Nuestros hallazgos indican la presencia de la mayoría de las conceptualizaciones, con predominio de aquellas que se desprenden de la definición analítica de pendiente como coeficiente paramétrico, razón algebraica y concepción trigonométrica y la que se aplica dentro de la misma geometría en la determinación del paralelismo o perpendicularidad entre rectas, como lo es la propiedad determinante. Estas conceptualizaciones, por un lado, inducen a la formación de la idea de que la pendiente tiene sentido sólo en el contexto intramatemático, y por otro lado, privilegian el desarrollo del conocimiento procedimental en detrimento del conocimiento conceptual. La comprensión de la pendiente requiere de la formación de redes internas como producto de conexiones entre conceptualizaciones en el plano intra y extra matemático, además del desarrollo armónico del conocimiento conceptual y el procedimental. Lograr la comprensión de los conceptos es fundamental en la Educación Matemática, sin embargo, nuestros resultados indican que los textos que utilizan los profesores difícilmente pueden contribuir a este logro.
\end{abstract}

Palabras clave: Libros de texto de matemáticas. Conceptualizaciones de pendiente. Bachillerato. Comprensión matemática.

\begin{abstract}
This paper reports the results of an investigation whose objective is to find which slope conceptualizations have a presence in high school mathematics textbooks and which are the predominant ones. For this, we used the Content Analysis method, where the objects of analysis are found in content exposition, worked examples, and in the exercises or problems proposed in the textbooks. As a reference framework we used the eleven slope conceptualizations identified by Stump (1999) and Moore-Russo, Conner and Rugg (2011). Our findings indicate the presence of most of the conceptualizations identified in the previous research, however, there is a notable predominance of those that emerge from the analytical definition of slope, such as the parametric coefficient,

\footnotetext{
* Doctor en Ciencias por el ISPEJV de La Habana, Cuba, profesor del Centro de Investigación en Matemática Educativa (CIMATE) de la Facultad de Matemáticas de la Universidad Autónoma de Guerrero, México. Dirección postal: Av. Lázaro Cárdenas S/N, Ciudad Universitaria, CP 39087, Chilpancingo, Gro., México. E-mail: cdolores2@gmail.com; cdolores@uagro.mx.

** Maestro en Ciencias con Especialidad en Matemática Educativa por la Universidad Autónoma de Guerrero. Estudiante de Doctorado en el Centro de Investigación en Matemática Educativa (CIMATE). Dirección postal: Av. Lázaro Cárdenas S/N, Ciudad Universitaria, CP 39087, Chilpancingo, Gro., México. E-mail: ibanez.dg@gmail.com; gibanez@uagro.mx.
} 
algebraic ratio, and trigonometric conception and its internal application in determination of parallelism or perpendicularity between lines as is the determining property. These conceptualizations, on the one hand, induce to formation of the idea that slope makes sense only in intra-mathematical context, and on the other hand, they favor the development of procedural knowledge on detriment of conceptual knowledge. Understanding the slope requires the creation of internal networks as a product of connections between conceptualizations intra and extra mathematical plane, in addition to the harmonious development of conceptual and procedural knowledge. Achieving the understanding of the concepts is essential for Mathematics Education, however, our results indicate that the texts used by teachers can hardly contribute to this achievement.

Keywords: Mathematics textbooks. Slope conceptualizations. High School level. Mathematics understanding.

\section{Introducción}

La pendiente es un concepto importante en la Educación Matemática porque describe el comportamiento de las funciones más básicas (TEUSCHER; REYS, 2010), es fundamento de conceptos matemáticos más avanzados como la derivada (DENIZ; KABAEL, 2017); y de conceptos estadísticos esenciales, como la regresión lineal y la línea de mejor ajuste (CASEY; NAGLE, 2016; NAGLE; CASEY; MOORE-RUSSO, 2017). Sin embargo, varias investigaciones han reportado muchas dificultades asociadas a su comprensión (v.g., CHO; NAGLE, 2017; WEBER; TALLMAN; MIDDLETON, 2015). Quizá una de las fuentes de tales dificultades sea la amplia variedad de formas en que se puede conceptualizar la pendiente.

El estudio de las conceptualizaciones de la pendiente proviene del interés por explorar el conocimiento y la comprensión de este concepto. La teoría de los concept image y concept definition de Tall y Vinner (1981) constituyó una base sólida para conocer la estructura cognitiva de los individuos acerca de este concepto. Sobre esta base y de la Teoría sobre el Conocimiento Pedagógico del Contenido de Shulman (1986), Stump (1999) identificó lo que a la postre se denominaron conceptualizaciones de la pendiente. Utilizando las conceptualizaciones de la pendiente se han realizado varios estudios que incluyen a estudiantes, profesores e, incluso, la currícula.

Por ejemplo, Nagle et al. (2013) descubrieron que estudiantes de cálculo que iniciaban la universidad utilizaban más la conceptualización de indicador de comportamiento, mientras que los profesores preferían utilizar la pendiente como una propiedad funcional. Casey y Nagle (2016) notaron que la desconexión entre las conceptualizaciones, razón geométrica y razón algebraica con la propiedad funcional afecta la comprensión del concepto de pendiente. S

Stanton y Moore-Russo (2012) analizaron la currícula de USA cifrada en los estándares de la NCTM (2000) y descubrieron en ellos que las conceptualizaciones más comunes de pendiente son: la razón geométrica, el indicador de comportamiento, la propiedad determinante, la propiedad física y la razón algebraica. Por su parte Dolores, Rivera y Moore-Russo (2020a), 
a diferencia de la currícula de USA, notaron presencia muy escasa de la razón geométrica en la currícula mexicana. Sin embargo, las investigaciones publicadas sobre la pendiente en los libros de texto son muy escasas.

Se han publicado investigaciones en español que analizan los textos de matemáticas, pero atendiendo aspectos distintos a la pendiente. Por ejemplo, González y Sierra (2004) estudiaron la presentación del contenido relativo a los puntos críticos en textos de análisis del preuniversitario español; Cordero, Cen y Suárez (2010) estudiaron los funcionamientos y formas de las gráficas en los libros de texto de bachillerato; Cordero y Flores (2007) estudiaron el uso de las gráficas en el discurso matemático escolar de los textos mexicanos del nivel básico.

Cercano a los estudios sobre la pendiente, se encuentra el trabajo de Hong y Choi (2018), quienes compararon el tratamiento de funciones lineales entre dos textos coreanos y uno estadounidense y encontraron que, este último, pone énfasis en las aplicaciones a la vida real de las funciones lineales en lugar de las matemáticas puras y los algoritmos matemáticos.

Choy, Lee y Mizzi (2015) examinaron las conceptualizaciones de la pendiente en textos de matemáticas de $8^{\circ}$ grado de Alemania, Singapur y Corea del Sur. Hacen notar, primero, que no presentan concepciones trigonométricas y de cálculo por ser nociones más avanzadas; segundo, las conceptualizaciones más variadas de la pendiente aparecen en el texto alemán con énfasis en el modelado, y tercero, notan una transición más estructurada en las conceptualizaciones tanto para Singapur como para Corea del Sur, donde la pendiente es parte del tema de la recta.

En el presente trabajo se estudian las conceptualizaciones de la pendiente en los libros de texto, pero utilizados en un contexto geográfico más limitado, situado en la ciudad capital del Estado de Guerrero, ubicado en el sur de México.

En las últimas décadas se ha logrado un progreso importante en la investigación sobre los libros de texto de matemáticas, el mayor logro se ha concentrado en el análisis de libros de texto (incluida la comparación entre ellos) y su uso en la enseñanza y el aprendizaje (FAN; ZHU; MIAO, 2013). Estos mismos autores proponen cinco direcciones para avanzar en la investigación en este campo: (1) contenido y temas de matemáticas; (2) cognición y pedagogía; (3) género, etnicidad, equidad, cultura y valor; (4) comparación de diferentes libros de texto; y (5) conceptualización y cuestiones metodológicas. El presente estudio se enmarca en la primera dirección, ya que estudia el contenido asociado a la pendiente a través de sus conceptualizaciones. Por ello, nos planteamos en este trabajo dar respuesta a una pregunta central: ¿qué conceptualizaciones de pendiente tienen presencia en los textos de matemáticas del bachillerato y cuáles son las predominantes? 


\section{Marco referencial}

\subsection{El libro de texto}

El libro de texto se define como un recurso impreso, publicado y diseñado para ser utilizado por maestros y alumnos, por lo que juega un papel central en el proceso de enseñanza y aprendizaje (VAN STEENBRUGGE; VALCKE; DESOETE, 2013; REMILLARD, 2005). Valverde et al. (2002, p. 2) explicaron, además, que "los libros de texto están diseñados para traducir las abstracciones de la política curricular en operaciones que los docentes y los estudiantes pueden llevar a cabo. Están pensados como mediadores entre las intenciones de los diseñadores de la política curricular y los maestros que imparten instrucción en las aulas".

Los libros de texto juegan un papel importante en la Educación Matemática, porque las oportunidades de aprendizaje para los estudiantes (en cuanto a dominio del contenido o de las habilidades cognitivas) se proporcionan justamente en los en libros de texto o documentos curriculares (FLODEN, 2002). Además, porque los libros de texto proporcionan explicaciones y ejercicios para que los estudiantes completen sus tareas de enseñanza y a los profesores les ofrecen guías de instrucción para orientar este proceso (VAN STEENBRUGGE; VALCKE; DESOETE, 2013). Por tanto, analizar las conceptualizaciones de pendiente en los textos puede dar información acerca de cómo están contribuyendo a la comprensión de este importante concepto en la Educación Matemática.

\subsection{Acerca de la pendiente}

Lehmann (1980, p. 17) define a la pendiente de una recta como coeficiente angular, la designa como " $m=\operatorname{tg} \alpha$, si pasa por los puntos $\mathrm{P}_{1}\left(x_{1}, y_{1}\right)$ y $\mathrm{P}_{2}\left(x_{2}, y_{2}\right)$ entonces $m=\frac{y_{1}-y_{2}}{x_{1}-x_{2}}, x_{1} \neq$ $x_{2}$ ”. Esta definición analítica está referida a la recta como lugar geométrico, en ella se destaca el ángulo de inclinación, su liga con la tangente trigonométrica y la razón geométrica en términos de la fórmula algebraica para calcular la pendiente. Sin embargo, la pendiente, además de ser un ente geométrico, tiene naturaleza variacional, según Thompson y Carlson (2017), porque enfatiza la razón entre los cambios de $y$ respecto de $x$, en referencia a las funciones cuya naturaleza está ligada a la covariación. Puede decirse que la pendiente es un caso particular de la razón de cambio, por ello se dice que la pendiente de una función lineal es la forma más 
básica de razón de cambio que encontrará un estudiante (STANTON; MOORE-RUSSO, 2012; TEUSCHERS; REYS, 2012).

Dada su naturaleza geométrica y variacional, mediante la pendiente se puede obtener la medición de la inclinación de una recta o el cambio en una función a través de una razón (ratio) o tasa (rate). Sin embargo, estos términos ocasionan confusiones, ya que no hay una distinción convencional entre ellos, afirman Thomson y Thompson (1992). Para Vergnaud (1983) una razón es una comparación entre cantidades de naturaleza similar y una tasa es una comparación de cantidades de naturaleza diferente. Para Ohlsson (1988), una razón es una expresión numérica de cuánto hay de una cantidad en relación con otra cantidad; una tasa es una relación entre una cantidad y un período de tiempo.

Sobre la base de las operaciones mentales, Thompson (1990) propuso una definición que resuelve esta confusión, plantea que una razón es una comparación multiplicativa entre dos cantidades, la cual enfatiza la relación cuantitativa de una relación que permite determinar cuántas veces mayor es una cantidad que otra. Por ejemplo, si la pendiente de una recta es $m=$ 2 , significa que el cambio en $y$ es dos veces más grande que el cambio en $x$; decir que un auto rinde $20 \mathrm{~km} /$ litro significa que la medida de la distancia respecto cantidad de combustible que consume está en razón de 20 a 1, es decir, es 20 veces más grande la distancia respecto de la unidad de volumen del combustible. Si las cantidades comparadas tienen el mismo atributo (v. gr., longitud), entonces es como abordar la pregunta ¿Cuántas veces es más grande esto que aquéllo?"; si las cantidades tienen diferentes atributos, entonces son sus segmentaciones las que se comparan (THOMPSON; THOMPSON, 1992, p. 6).

El concepto de pendiente está presente tanto al interior del aula escolar como fuera de ella. En el curriculum mexicano, del estudio de la razón de cambio emerge el concepto de pendiente (, 2020a). La razón de cambio (promedio) se define en Stewart (2012, p.147-148) como "el cociente de diferencia $\frac{\Delta y}{\Delta x}=\frac{f\left(x_{2}\right)-f\left(x_{1}\right)}{x_{2}-x_{1}}$ en el intervalo $\left[x_{1}, x_{2}\right]$ ". Esta expresión en términos gráficos se interpreta como la pendiente de la secante a la curva $f$. Sin embargo, su significado más rico se encuentra en la medición del cambio en situaciones del mundo real.

Estas existen en dos formas diferentes: a) situaciones físicas estáticas, como las pistas de esquí y rampas para sillas de ruedas, y b) situaciones funcionales dinámicas, tales como distancia versus tiempo o cantidad versus costo (STUMP, 2001). El cambio se calcula mediante las diferencias: $\Delta x=x_{2}-x_{1}$ y $\Delta y=f\left(x_{2}\right)-f\left(x_{1}\right)$; por lo tanto, se puede decir que $\frac{\Delta y}{\Delta x}$ es una razón entre cambios. Si esa razón es una constante, entonces se trata de una función lineal de 
la forma $f(x)=m x+b$, donde $m=\frac{\Delta y}{\Delta x}$. Lo constante de la razón de cambio es una característica esencial de la linealidad y de los modelos lineales.

La razón de cambio tiene diferentes denominaciones dependiendo del contexto. En Cinemática suele utilizarse como velocidad $(v)$ o aceleración $(a)$, la primera mide la variación de la distancia respecto del tiempo, la segunda cuantifica la variación de la velocidad respecto del tiempo. En Hidráulica se utiliza como Gasto (Q), se define como la cantidad o volumen de fluído que pasa por un ducto en un intervalo de tiempo. En Demografia suele utilizarse como tasa de crecimiento poblacional (TCD), tasa de natalidad o de mortalidad, que dan cuenta de la variación (aumento o disminución) de la población respecto del tiempo o de la cantidad de nacimientos o defunciones en un intervalo de tiempo. En cualquiera de los casos, se trata de razones de cambio o razones entre cambios. Si esas razones son constantes, se obtienen de modelos lineales cuyas gráficas son lineas rectas y su pendiente es la misma en cualquier punto. Si las razones de cambio son variables, se obtienen de funciones cuyas gráficas son curvas y sus pendientes también son variables.

\subsection{Las conceptualizaciones de la pendiente}

En el proceso de formación de conceptos matemáticos tienen lugar las imágenes conceptuales, y estas se entienden como la estructura cognitiva total que se asocia con un concepto dado (TALL; VINNER, 1981), se hacen visibles a través de representaciones simbólicas, imágenes, tablas, lenguaje oral o escrito. Una representación se conforma por todas aquellas herramientas signos o gráficos que hacen presente el concepto y los procedimientos matemáticos, con las cuales cada sujeto aborda e interactúa con el conocimiento matemático (HOFFMAN, 2015).

En este trabajo asumimos la concepción de Hoffman, la cual considera a la conceptualización de la pendiente como una representación específica de este concepto. Por

ejemplo, la representación $\frac{y_{2}-y_{1}}{x_{2}-x_{1}}$, es la fórmula algebraica para obtener la pendiente de una recta, es la base de los procedimientos para calcularla y está ligada a la conceptualización razón algebraica (ver Cuadro 1). Los libros de texto utilizan representaciones variadas en la exposición del tema, en los ejemplos, en los ejercicios o problemas propuestos, para dar oportunidad a que los estudiantes se formen conceptos, realicen procedimientos y puedan resolver problemas. Al hablar de una representación específica de la pendiente, nos referimos a alguna de las conceptualizaciones descritas en el Cuadro 1. 


\begin{tabular}{|c|c|c|}
\hline Categoría & La pendiente como... & Código \\
\hline Razón geométrica & $\begin{array}{l}\text { Razón del desplazamiento vertical sobre el desplazamiento horizontal en la } \\
\text { gráfica de una recta (a menudo vista como el triángulo rectángulo en la gráfica } \\
\text { de una recta que resalta el desplazamiento horizontal y el vertical). }\end{array}$ & G \\
\hline Razón algebraica & $\begin{array}{l}\text { Cambio en } y \text { sobre el cambio en } x \text {; representación de la razón con expresiones } \\
\left.\text { algebraicas (a menudo visto como } \frac{\Delta y}{\Delta x} \circ \frac{y_{2}-y_{1}}{x_{2}-x_{1}}\right) \text {. }\end{array}$ & A \\
\hline Propiedad física & $\begin{array}{l}\text { Propiedad de la recta a menudo descrita con expresiones como: grado, } \\
\text { inclinación, ladeo, declive, empinado, qué tan alto sube una recta. }\end{array}$ & $\mathrm{P}$ \\
\hline $\begin{array}{l}\text { Propiedad } \\
\text { funcional }\end{array}$ & $\begin{array}{l}\text { Razón de cambio constante entre variables o cantidades (por ejemplo, cuando } \\
x \text { aumenta } 2, y \text { aumenta } 3 \text { ) presente en varias representaciones de funciones; a } \\
\text { veces se vé en situaciones que involucran razones relacionadas o constantes de } \\
\text { proporcionalidad (donde la tasa unitaria es la pendiente). }\end{array}$ & $\mathrm{F}$ \\
\hline $\begin{array}{l}\text { Coeficiente } \\
\text { paramétrico }\end{array}$ & $\begin{array}{l}\text { Coeficiente } m \text { (o su valor numérico) en } y=m x+b \text { o en } \\
y-y_{1}=m\left(x_{2}-x_{1}\right) \text { que actúa como un parámetro. }\end{array}$ & $\mathrm{PC}$ \\
\hline $\begin{array}{l}\text { Concepción } \\
\text { trigonométrica }\end{array}$ & $\begin{array}{l}\text { Propiedad relacionada con el ángulo que una recta forma con una recta } \\
\text { horizontal (usualmente el eje positivo } x \text { ); tangente del ángulo de inclinación. }\end{array}$ & $\mathrm{T}$ \\
\hline $\begin{array}{l}\text { Concepción en } \\
\text { Cálculo }\end{array}$ & $\begin{array}{l}\text { Medida relacionada con la derivada como la pendiente de la tangente a una } \\
\text { curva, de una recta secante, o cómo razón de cambio instantánea para } \\
\text { cualquier función (incluso no lineal). }\end{array}$ & $\mathrm{C}$ \\
\hline $\begin{array}{l}\text { Situación del } \\
\text { mundo real }\end{array}$ & $\begin{array}{l}\text { Situación física (estática, por ejemplo: una rampa, escalera etc.) } \\
\text { Situación funcional dinámica que relaciona dos variables (v.g., distancia } v s \\
\text { tiempo, velocidad } v s \text { tiempo, costo } v s \text { tiempo). }\end{array}$ & $\mathrm{R}$ \\
\hline $\begin{array}{l}\text { Propiedad } \\
\text { determinante }\end{array}$ & $\begin{array}{l}\text { Propiedad que determina si las rectas son paralelas o perpendiculares entre sí; } \\
\text { propiedad con la cual una recta puede ser determinada si un punto de la recta } \\
\text { es también dado. }\end{array}$ & $\mathrm{D}$ \\
\hline Constante lineal & $\begin{array}{l}\text { Recta o plana, ausencia de curvatura de una recta que no se ve afectada por la } \\
\text { traslación; propiedad única de las rectas (puede ser referenciada como lo que } \\
\text { hace que una línea sea recta o la rectitud de una línea); mención de que } \\
\text { cualesquiera dos puntos de una recta pueden ser utilizados para determinar la } \\
\text { pendiente. }\end{array}$ & $\mathrm{L}$ \\
\hline $\begin{array}{l}\text { Indicador de } \\
\text { comportamiento }\end{array}$ & $\begin{array}{l}\text { Propiedad que indica el crecimiento, decrecimiento, tendencia horizontal de } \\
\text { una recta o la propiedad que indica la cantidad (o severidad) del aumento o } \\
\text { disminución de una recta; si no es cero, indica que la recta tiene una } \\
\text { intersección con el eje } x \text {. }\end{array}$ & B \\
\hline
\end{tabular}

Cuadro 1 - Conceptualizaciones de la pendiente

Fuente: Adaptado de Nagle y Moore-Russo (2013c)

\section{Método}

El análisis de los textos se realizó utilizando el método de Análisis de Contenido sugerido por Bardin (2002, p. 87), consta de tres fases: pre-análisis, explotación del material y el tratamiento de los resultados. En la fase de pre-análisis, se preparó el material, consistente en la identificación y elección de los libros de texto. Estos fueron elegidos sobre la base de tres criterios: los que tratan de manera formal la pendiente, los que son recomendados por la currícula oficial y los que más utilizan los profesores.

Según la currícula, este concepto es introducido por primera vez en el $9^{\circ}$ grado y recibe un tratamiento más amplio y formal en el grado $11^{\circ}$ (DOLORES et al., 2020), grado en el que se imparte el curso de Matemáticas III. Por tanto, nos centramos en los libros texto que más 
utilizan para impartir este curso. Para saberlo, se aplicó una encuesta a veinte profesores de matemáticas de la región, preguntándoles qué textos más utilizaban de los previstos en la currícula oficial. Según sus respuestas, estos textos son: Fuenlabrada y Fuenlabrada (2014), (GA); Méndez (2013), (MIII) y Salazar (2018), (M3).

La fase de explotación del material está centrada en las operaciones de codificación, elección de las unidades de análisis y análisis del contenido. Adoptamos la codificación y caracterización de las conceptualizaciones de pendiente de Nagle y Moore-Russo (2013c), ya descritas en el Cuadro 1. Las unidades de análisis se obtuvieron de la estructura de los libros de texto, en general el contenido se estructura de: (1) exposición del tema, (2) ejemplos resueltos y (3) ejercicios o problemas propuestos.

En el primero se explican conceptos teóricos, que incluyen diagramas, gráficas, definiciones, fórmulas o teoremas; el segundo contiene problemas o ejercicios resueltos junto con una explicación de su solución; en el tercero están los problemas o ejercicios propuestos, los cuales se espera que los estudiantes resuelvan. Los contenidos de los textos analizados corresponden a un curso clásico de Geometría Analítica y se refieren a: Ecuación de la recta, la Circunferencia, la Parábola, la Elipse, la Hipérbola y, eventualmente, la Ecuación General de las cónicas. Las unidades específicas de análisis fueron las frases, procedimientos o términos claves que hacen referencia a alguna de las conceptualizaciones de la pendiente descritas en el Cuadro 1.

Para la recolección de datos se elaboró una matriz de conceptualizaciones $v s$ frases. Las primeras se ubicaron en once columnas y en cada renglón se ubicaron las frases tomadas de los libros de texto en donde se localizaban las conceptualizaciones buscadas. Los ejercicios o problemas propuestos fueron resueltos por los investigadores, considerando la información dada en la exposición del tema respectivo, esto con el fin de desentrañar las conceptualizaciones utilizadas. En el Cuadro 2 damos algunos ejemplos de cómo se identificaron.

\begin{tabular}{|c|c|c|}
\hline Frases & Argumentos & $\begin{array}{l}\text { Conceptua- } \\
\text { lizaciones }\end{array}$ \\
\hline $\begin{array}{l}\text { Dos rectas son perpendiculares si la pendiente } \\
\text { de una de ellas es la recíproca negativa de la } \\
\text { pendiente de la otra.. Se sabe que: } \\
\text { tan }\left(90^{\circ}+\theta_{1}\right)=\cot \theta_{1} \text {. Sustituyendo el } \\
\text { valor de } \theta_{2}, \tan \theta_{2}=-\cot \theta_{1}=\frac{-1}{\tan \theta_{1}} \text {. } \\
\text { Expresando esto en términos algebraicos } \\
\text { queda: } m_{2}=-\frac{1}{m_{1}} \text {. } \\
\text { Fuenlabrada y Fuenlabrada }(2014, \text { p. } 35-36) \text {. }\end{array}$ & $\begin{array}{l}\text { La condición de perpendicularidad indica la } \\
\text { presencia de la conceptualización } \\
\text { propiedad determinante. } \\
\text { Las expresiones del tipo: } \tan \left(90^{\circ}+\theta_{1}\right) \\
\text { indican la presencia de la concepción } \\
\text { trigonométrica. } \\
\text { En la expresiones } m_{2}=-\frac{1}{m_{1}} \text { está presente } \\
\text { la conceptualización coefiente paramétrico. }\end{array}$ & $\mathrm{D}, \mathrm{T}, \mathrm{PC}$ \\
\hline $\begin{array}{l}\text { Por ejemplo: Si la recta pasa por los puntos } \\
A(-1,6) \text { y } m=-4 \text {, entonces su ecuación }\end{array}$ & $\begin{array}{l}\text { El uso de las variables } m \text { y su valor } \\
\text { numérico evidencían la presencia de la } \\
\text { conceptualización coeficiente paramétrico. }\end{array}$ & PC, G. \\
\hline
\end{tabular}




\begin{tabular}{|c|c|c|}
\hline $\begin{array}{l}\text { corresponde a: } \quad y-y_{1}=m\left(x-x_{1}\right) \text {. } \\
\text { Sustituyendo valores: } \\
\quad y-6=-4[x-(-1)] \\
\quad y-6=-4[x+1] \\
\text { Esta es la forma de representar la recta pero ... } \\
\text { Esta es la forma más común que aparece: } \\
y-6=-4 x-4 \\
y=-4 x-4+6 \\
y=-4 x+2 \\
4 x+y+2=0 \\
\text { Ecuación lineal con } \\
\text { dos variables. } \\
\text { Salazar (2018), p. } 80\end{array}$ & $\begin{array}{l}\text { La representación en la gráfica del } \\
\text { desplazamiento en } x \text { e } y \text { para representar la } \\
\text { pendiente denota la utilización de la } \\
\text { conceptualización razón geométrica. }\end{array}$ & \\
\hline $\begin{array}{l}\text { "Una empresa recibe un préstamo por } \$ 120000 \\
\text { con un interés simple de } 4 \% \text { sobre el capital } \\
\text { inicial. Haz la gráfica y determina el valor de la } \\
\text { pendiente". }\end{array}$ & $\begin{array}{l}\text { Es una situación funcional dinámica porque } \\
\text { relaciona las variables: dinero y tiempo, por } \\
\text { tanto utiliza la conceptualización del } \\
\text { mundo real. } \\
\text { Está presente la razón de cambio constante } \\
\text { en el interés simple, por tanto puede } \\
\text { utilizarse la conceptualización propiedad } \\
\text { funcional. La construcción de la gráfica } \\
\text { puede inducir al uso de la razón geométrica } \\
\text { y el cálculo de la pendiente al uso del } \\
\text { coeficiente paramétrico. }\end{array}$ & $\mathrm{R}, \mathrm{F}, \mathrm{G}, \mathrm{PC}$ \\
\hline
\end{tabular}

Cuadro 2 - Ejemplos de conceptualizaciones sobre la pendiente en los textos analizados Fuente: adaptación de los autores, sin pérdida de la esencia planteada en los textos analizados

En la tercera fase, dedicada al tratamiento de los resultados, se empleó al método de revisión por pares. Para ello, de forma independiente, el equipo de investigación revisó los textos y clasificó las conceptualizaciones encontradas. Primero, se leyó cada texto con el objetivo de encontrar referencias a la pendiente. Posteriormente, se buscaron las frases clave contenidas en el Cuadro 1, de manera que permitieran la categorización de los contenidos y para finalizar en conjunto se compararon y discutieron las clasificaciones propuestas. En caso de algún desacuerdo, los investigadores discutieron el extracto hasta llegar a un consenso sobre el tipo de conceptualización presente. Finalmente, para determinar la frecuencia de las conceptualizaciones, se consideró el número de referencias encontradas en cada uno de ellos y posteriormente se elaboró una matriz general que incluyó los tres textos en conjunto.

\section{Resultados}

\subsection{Conceptualizaciones en el texto GA}

En este texto las conceptualizaciones fueron encontradas en los temas: Ecuación de la línea Recta y Casos Especiales, Ecuación de la Circunferencia, Parábola, Elipse, Hipérbola y en la Ecuación General de las Cónicas. La mayor parte se concentra en el primero, sólo en el 
tratamiento de este tema identificamos 321, de un total de 420. Destacan las conceptualizaciones: coeficiente paramétrico, propiedad determinante y razón algebraica, todas ellas utilizadas en los ejercicios propuestos (ver Figura 1). Es notable la utilización de procedimientos algebraicos tanto en la exposición del tema, como en los ejemplos resueltos o en los ejercicios y problemas propuestos, en esta última parte encontramos expresiones del tipo: " $m=\frac{y_{2}-y_{1}}{x_{2}-x_{1}}$ es la relación para obtener la pendiente de la recta que pasa por los puntos $\mathrm{A}\left(x_{1}\right.$, $\left.y_{1}\right)$ y $\mathrm{B}\left(x_{2}, y_{2}\right)$ " (FUENLABRADA; FUENLABRADA, 2014, p. 33). "Si el ángulo de inclinación de una recta es de 52, ¿cuál es su pendiente?” (p. 35). “Obtenga la pendiente de la recta que pasa por los puntos $(1,-3)$ y $(4,1)$ ” (p. 52). "Determina si la recta que pasa por los puntos $(6,0),(0,4)$ y la que pasa por $(0,2),(3,0)$ son paralelas" (p. 36). "Obtenga la ecuación de la recta con pendiente $m=-\frac{1}{2}$ y que pasa por el punto $(-2,3)$ " (p. 48).

En el tema Ecuación de la Circunferencia las conceptualizaciones de la pendiente se concentran en las relaciones que se establecen entre la circunferencia y sus tangentes. Aquí se notan las conceptualizaciones coeficiente paramétrico y propiedad determinante, las cuales derivan de problemas tales como Obtenga la ecuación la recta tangente a la circunferencia $x^{2}+y^{2}=25$ con $m=-3 / 4$. En el tema de la Parábola y la Elipse las conceptualizaciones se concentran en la obtención de los puntos de intersección de las rectas secantes con estas curvas; en el tema de la Hipérbola se hacen evidentes en la obtención de las ecuaciones de sus asíntotas. En el tema Ecuación General de las Cónicas las conceptualizaciones están presentes en las aplicaciones de las ecuaciones de las cónicas a la industria y a la ingeniería, aquí utilizan la conceptualización propiedad funcional conectando la pendiente y la razón de cambio, además se insiste en la ecuación de la tangente a la circunferencia. 


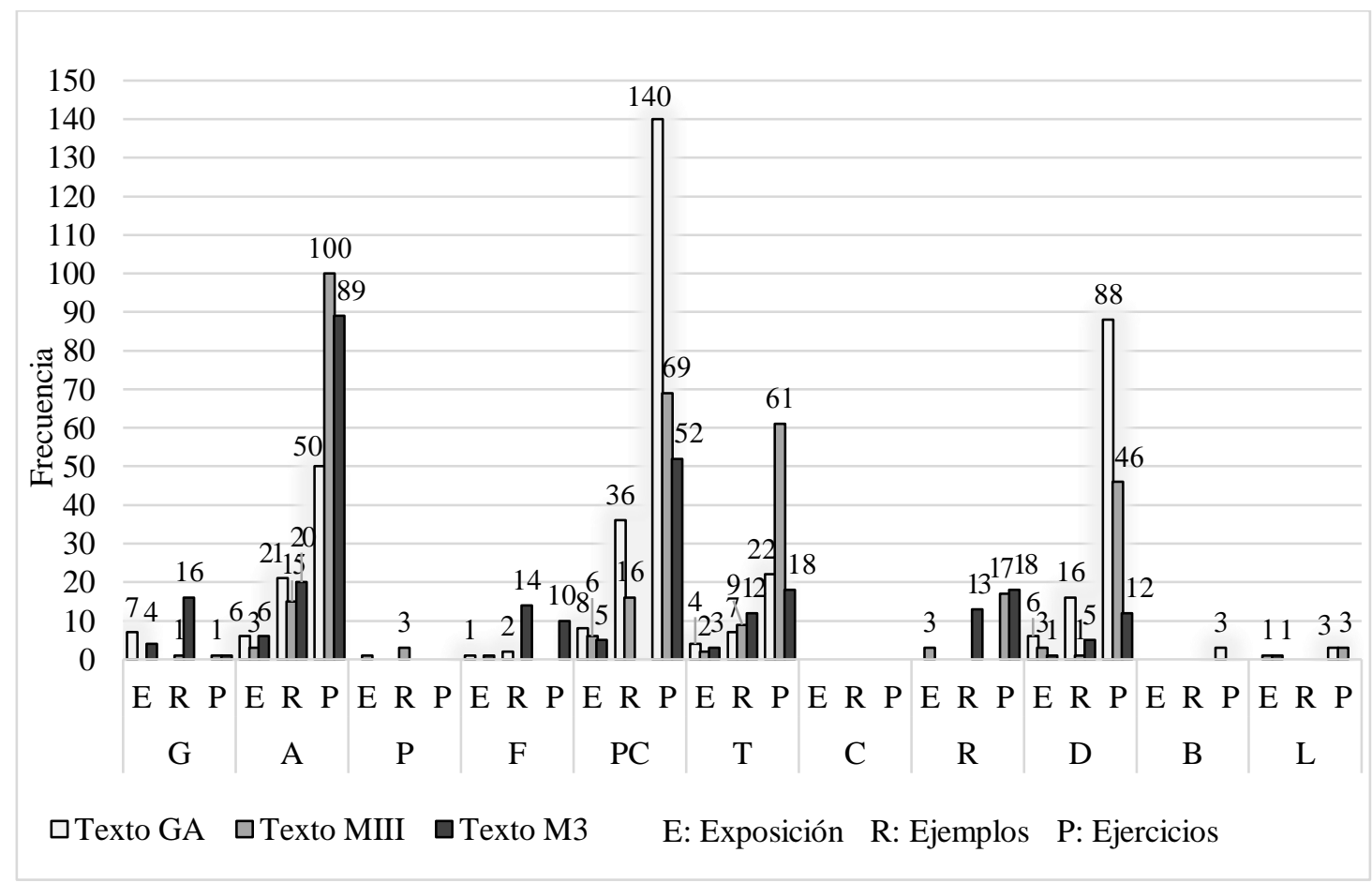

Figura 1 - Conceptualizaciones de la pendiente en los tres textos Fuente: elaboración propia a partir de los datos obtenidos del análisis del contenido

De las cuatro conceptualizaciones predominantes en este texto, tres están directamente relacionadas con la definición analítica de pendiente: coeficiente paramétrico, razón algebraica y la concepción trigonométrica (ver Figura 1). La restante conceptualización, propiedad determinante, que ocupa el tercer lugar en frecuencia, tiene que ver con la relación entre dos rectas, la de paralelismo y perpendicularidad.

En este texto es escasa la presencia de la conceptualización razón geométrica, a pesar de que utiliza con frecuencia las gráficas de rectas y enfatiza los puntos por donde pasa, los puntos de corte con los ejes $x$ e $y$, escasamente utiliza los catetos de los triángulos rectángulos para el cálculo de las diferencias: $y_{2}-y_{1}$ y $x_{2}-x_{1}$ para determinar la pendiente, de hecho la idea de lo que sube entre lo que avanza no se menciona en este texto. No encontramos evidencia de la presencia, en este texto, de las conceptualizaciones propiedad física, concepción en Cálculo y situación del mundo real

\subsection{Conceptualizaciones en el texto MIII}

En este texto todas las conceptualizaciones identificadas están concentradas en el tema de la línea recta. En el tratamiento de este tema, identificamos 361 conceptualizaciones. Observamos predominio de las conceptualizaciones: razón algebraica, coeficiente paramétrico, concepción trigonométrica y propiedad determinante, localizadas en los ejercicios propuestos 
(ver Figura 1) en los cuales se requiere la utilización de procedimientos algebraicos al aplicar las distintas formas de la ecuación de la recta. En ese sentido plantean problemas del tipo: "Encuentra la ecuación de la recta que pasa por los puntos siguientes $(-8,9)$ y $(6,-6)$ " (Méndez 2013, p. 73). "Encuentra la pendiente y el ángulo de inclinación de la recta que pasa por los puntos $(7,9),(-6,-5)$ " (p. 54). "Indica si las rectas son perpendiculares o paralelas: $l_{1} A(-2$, $-5) ; B(4,7) ; l_{2} P(-3,-3) ; Q(4,11)$ " (p. 61).

A diferencia del texto GA, en MIII tienen presencia las conceptualizaciones relativas al mundo real, encontramos veinte de ellas, principalmente las asociadas a la situación funcional. Se nota interés en establecer conexiones entre pendiente con problemas o situaciones de la física o de la economía, por ejemplo, plantea situaciones de cuerpos en movimiento denotando que el movimiento uniforme está relacionado con la velocidad o que los fenómenos de oferta y demanda pueden ser representados mediante rectas en el plano cartesiano y que están asociadas a la pendiente. Las conceptualizaciones: constante lineal, propiedad física y razón geométrica son las de menor presencia. No encontramos evidencia de las conceptualizaciones propiedad funcional, indicador de comportamiento y concepción en Cálculo.

\subsection{Conceptualizaciones en el texto M3}

Prácticamente todas las conceptualizaciones en este texto están concentradas en el tema de la línea recta, algunas están presentes en el tema de la circunferencia, están ausentes en los demás contenidos. En el tema de la línea recta identificamos 263 conceptualizaciones y doce en el tema de la circunferencia. Al igual que en los dos textos anteriores, parte importante de las conceptualizaciones son utilizadas en los procedimientos algebraicos asociados a las formas de la ecuación de la recta.

A diferencia de los dos textos anteriores, en M3 encontramos veinte conceptualizaciones del tipo razón geométrica (ver Figura 1) donde se hace explícito lo que sube entre lo que avanza, además de la utilización de los catetos de triángulos rectángulos para indicar las diferencias $y_{2}-y_{1}$ y $x_{2}-x_{1}$. También, en este texto (igual que en MIII) identificamos una cantidad significativa de conceptualizaciones relativas al mundo real, 31 en total, y catorce de la propiedad funcional (ver Figura 1). Respecto de la primera, propone situaciones del tipo - Un contenedor de aceite de 100 litros tarda en llenarse 5 minutos, expresa el volumen $V$ en términos del tiempo $t$, ¿Qué significa la pendiente en este contexto? 
En este texto, en total, identificamos 301 conceptualizaciones, siendo las más diversificadas que en los otros dos, pero concentradas en el tema de la ecuación de la recta. En este tema, tres conceptualizaciones predominan: razón algebraica, coeficiente paramétrico y concepción trigonométrica, concentradas en los ejercicios propuestos (ver Figura 1). Aunque con menos frecuencia, están las conceptualizaciones: propiedad física, situación del mundo real, la razón geométrica y propiedad determinante. No encontramos evidencia de las conceptualizaciones: indicador de comportamiento, concepción en cálculo y propiedad física.

\section{Discusión}

Los resultados globales indican la presencia de diez conceptualizaciones de pendiente (ver Figura 2). La ausencia de la concepción en Cálculo se justifica porque está reservada para ser tratada en $12^{\circ}$ grado cuando se cursa Cálculo. Cuatro son las conceptualizaciones dominantes en los textos, la mayoría está concentrada en los ejercicios propuestos (ver Figura 2), son las que se desprenden de la definición analítica de pendiente: coeficiente paramétrico, razón algebraica, concepción trigonométrica y la relacionada con sus aplicaciones dentro de la propia geometría, la propiedad determinante. Todas ellas dan altas oportunidades a los estudiantes de establecer conexiones sólo al interior de la misma matemática. Por el contrario, las vinculadas a las aplicaciones extramatemáticas, como la situación del mundo real, la propiedad funcional y la propiedad física, son escasamente utilizadas (ver Figura 2), a pesar de que la currícula recomienda la aplicación de la matemática a situaciones reales (GOBIERNO DE MÉXICO, SEP, 2008).

Se nota, particularmente en el texto GA, privilegio de los temas clásicos de la Geometría Analítica, en los textos MIII y M3, hay evidencias de más apertura al introducir conceptualizaciones asociadas al mundo real, en específico propician la modelación y análisis de las situaciones funcionales. Sin embargo, la correspondencia entre la currícula y los textos es una situación compleja. Al estudiar el discurso de los textos de matemáticas en relación a la currícula prevista en USA, Herbel-Eisenmann (2007) afirma que es difícil alcanzar los objetivos de la currícula, y que puede haber una falta de coincidencia entre este objetivo y los contenidos de libros de texto convencionales. Esta misma situación parece estar ocurriendo en México, por lo que merece más atención por parte de quienes diseñan la currícula y los autores de libros de texto para enfrentar el problema de esta falta de coincidencia.

El énfasis en las conceptualizaciones derivadas de la definición analítica puede inducir desconexiones en la mente de los estudiantes, entre la definición de pendiente (que es 
intramatemática) y las aplicaciones extramatemáticas, en particular con las aplicaciones de la razón de cambio a problemas del mundo real. Hay evidencia, en Dolores, Rivera y Garcia (2019), que al plantearles tareas a estudiantes de la misma región sobre la pendiente y las razones de cambio como la velocidad, rapidez y aceleración, consideraron la pendiente como desligada de éstos conceptos. Las causas podrían atribuirse no sólo a cuestiones cognitivas propias de los estudiantes, sino a que están siendo instruidos con textos que privilegian conceptualizaciones asociadas sólo a ideas intramatemáticas. Así lo insinúan nuestros datos.

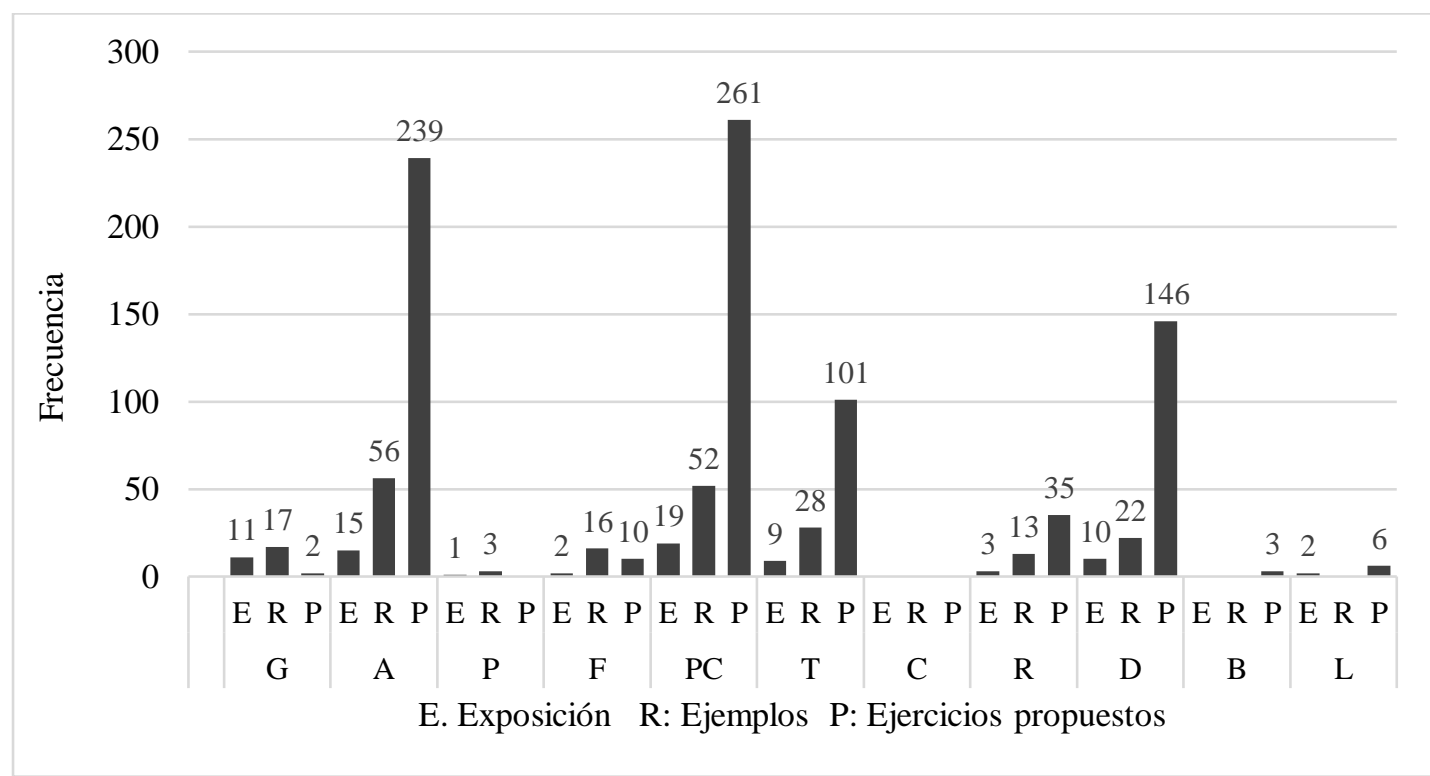

Figura 2 - Conceptualizaciones de la pendiente identificadas (total: 1082)

Fuente: elaboración propia a partir de los datos obtenidos del análisis del contenido

Varios investigadores (v.g., HIEBERT; CARPENTER, 1992; NOSS; HEALY; HOYLES, 1997; BERRY; NYMAN, 2003; BUSINSKAS, 2008; BARMBY et al., 2009; CAI; DING, 2015) señalan la necesidad de las conexiones en la comprensión de los conceptos matemáticos. Sin embargo, las ideas que se aprenden dentro de la matemática no se conectan, o transfieren de manera automática, a otros contextos.

Algunos investigadores demuestran que los conocimientos de los estudiantes sobre la pendiente no se transfieren entre los tipos de problemas (por ejemplo, cualitativo versus cuantitativo y matemático versus la configuración del mundo real), o incluso, del conocimiento de la matemática a la física (PLANINIC et al., 2012) y que además, los estudiantes no relacionan los conceptos de pendiente y razón de cambio (STUMP, 2001; LOBATO; SIEBERT, 2002; LOBATO; THANHEISER, 2002; TEUSCHER; REYS, 2010; DOLORES, et al., 2019).

Varias pueden ser las causas de la escasa transferencia y desconexión del conocimiento matemático, algunas pueden estar en el currículo previsto, otras pueden estar en los materiales 
y medios que usan los profesores, otras pueden estar en los métodos y estrategias didácticas utilizadas. También las dificultades intrínsecas al conocimiento mismo (SIERPINSKA, 1985; BROUSSEAU, 2002) o las dificultades cognitivas de los propios estudiantes pueden interferir el proceso. En esta complejidad, sin embargo, no se puede desdeñar la influencia que los textos ejercen sobre estudiantes y profesores. Nosotros creemos que las conexiones y la transferencia del conocimiento deben ser intencionalmente promovidas por los textos y los profesores. Pero las evidencias mostradas en este trabajo indican que esto no está ocurriendo así para la mayoría de las conceptualizaciones utilizadas en los textos analizados.

Las conceptualizaciones, coeficiente paramétrico y razón algebraica, también aparecen con la más alta frecuencia porque son de uso obligado tanto en la exposición del tema como en los ejemplos resueltos (ver Figura 2). Además, se utilizan tanto en las formas de la ecuación de la recta como en procedimientos necesarios al aplicarlas en los ejercicios o problemas que se esperan los estudiantes resuelvan. No obstante, su cuantiosa presencia puede inducir al desarrollo de un pensamiento procedimental más que el conceptual, así como lo conciben Hiebert y Lefevre (1986), Hiebert y Carpenter (1992) y Rittle-Johnson y Schneider, (2015). Por ejemplo, la conceptualización razón geométrica tiene escasa presencia en los textos (excepto en M3, ver Figura 1) pero tiene la potencialidad de desarrollar el pensamiento visual, en cambio proponen abundantes ejercicios cuya solución requiere de manipulaciones algebraicas en las que está presente la conceptualización coeficiente paramétrico.

Los textos analizados prestan más atención a los procedimientos para encontrar la pendiente que al desarrollo de las nociones conceptuales asociadas a este concepto, como sugieren Lingefjärd y Farahani (2018) y, con ello, sólo estimulan el pensamiento procedimental. En Dolores et al., (2019), se aporta evidencia que robustece esta hipótesis, al pedirles el cálculo de la pendiente a estudiantes de la misma región, casi dos tercios fueron capaces de obtenerla correctamente usando fórmulas y procedimientos algebraicos; en cambio, casi todos fueron incapaces de expresar un significado coherente sobre el resultado, o de plano dijeron no saber qué significaba ese resultado. Esto fue posible porque recordaron y usaron la fórmula para hacer procedimientos, priorizando los algoritmos, esto mismo se ha encontrado en otras investigaciones como las de Birgin (2012), Deniz y Kabael (2017) y Nagle y Moore-Russo (2013b). Sin embargo, la inclinación hacia lo procedimental no es exclusiva de los textos analizados, en otras latitudes Dole y Shield (2008) y Stacey y Vincent (2009) han detectado esta tendencia en textos de matemáticas australianos.

El tratamiento de la pendiente en los textos analizados se concentra en el tema de la ecuación de la recta, con escasas menciones en los temas de la circunferencia y la hipérbola 
como es el caso del texto GA, los otros dos textos exclusivamente se centran en la recta. Esto podría generar desconexión entre las conceptualizaciones de la pendiente asociadas a la recta y las que se pueden asociar a las curvas cónicas, ya que, dejan sin explotar las conexiones entre las cónicas y sus tangentes, una de las fuentes principales del concepto de derivada.

\section{Conclusiones y futuros trabajos}

Hemos identificado, en los textos, la presencia de diez de las conceptualizaciones previstas por Nagle y Moore-Russo (2013c), predominando en ellos: la del coeficiente paramétrico, la razón algebraica, la concepción trigonométrica y la propiedad determinante; las tres primeras provenientes de la definición analítica de pendiente y la última de sus aplicaciones al interior de la matemática. En conjunto, constituyen el $88.5 \%$ del total y propician el uso excesivo del lenguaje algebraico, de reglas y algoritmos, mediante la manipulación de expresiones tales como: $y=m x+b ; m=\frac{y_{2}-y_{1}}{x_{2}-x_{1}} ; m=\tan \theta, m_{1} m_{2}=-1$.

Las seis restantes, sólo constituyen el $11.5 \%$ del total y son las que ayudan a conectar la pendiente con el mundo real, a relacionarla con las propiedades de las funciones o con el mundo físico o a visualizarla como lo que sube entre lo que avanza. Pero estas conceptualizaciones son minimizadas en los textos. Este marcado contraste, cargado hacia las conceptualizaciones provenientes de la definición analítica induce, por un lado, a la formación de interpretaciones sólo intramatemáticas, y por otro lado, a privilegiar el desarrollo del conocimiento procedimental (el saber cómo) en menoscabo del conocimiento conceptual (el saber qué o por qué) y de la comprensión. El desarrollo del conocimiento conceptual es logrado mediante la construcción de relaciones entre piezas de información (HIEBERT; LEFEVRE, 1986, p. 4), sin embargo los textos siguen el sentido contrario, inducen la formación de escasas relaciones entre las conceptualizaciones de la pendiente por lo que en poco contribuyen a su comprensión.

Trabajos recientes, realizados en la misma región que el presente, fortalecen la afirmación anterior, los estudiantes son instruidos haciendo énfasis en las mismas conceptualizaciones que promueven los textos aquí analizados (RIVERA; SALGADO; DOLORES, 2020); incluso después de ser instruidos, las conceptualizaciones que más conocen son la propiedad física y la razón algebraica (RIVERA, et al., 2019) que inducen al conocimiento procedimental. Por tanto, el escaso conocimiento logrado por los estudiantes parece ser inducido por los textos utilizados y por las actividades trabajadas en clase, afectando, con ello, el desarrollo de su comprensión. 
Para Hiebert y Carpenter (1992), la comprensión matemática es como una red interna de representaciones de ideas, procedimientos y hechos matemáticos, en una dirección similar, pero para el concepto de pendiente en particular, Nagle y Moore-Russo (2013a) y Courtney, Martínez-Planell y Moore-Russo (2019) proponen un modelo de Red de Conceptualizaciones de la pendiente, que puede contribuir a una mejor comprensión de este concepto en el preuniversitario y formar las condiciones previas para que los estudiantes puedan comprender las ideas matemáticas del nivel superior.

Para contribuir a este propósito, los libros de texto debieran incluir una amplia y variada gama de conceptualizaciones que den oportunidad a los estudiantes de formar y desarrollar redes de conceptualizaciones. Estas les permitirían, por un lado, desarrollar sus habilidades en la resolución de problemas en diversos contextos, y por otro lado, preparar mejores condiciones de ingreso a sus estudios universitarios.

Los textos se consideran como el plan de estudios potencialmente implementado (SCHMIDT et al., 2001; VALVERDE et al., 2002). En este sentido, nuestro estudio no proporciona información acerca del currículum previsto (o planeado), del currículum implementado ni del currículo alcanzado. Varias investigaciones destacan las dificultades de los profesores en implementar el currículo (v.g., MANOUCHEHRI; GOODMAN, 1998; STUMP, 2001), otras investigaciones enfocan su atención sobre el currículo alcanzado (p.e. NAGLE; MOORE-RUSSO; STYERS, 2017).

Algunas investigaciones, como Remillard y Heck, (2014) y Gehrke, Knapp y Sirotnik, (1992), utilizan el término experienced para referirse al currículum que realmente toma lugar en el salón de clase mediante las interacciones entre profesores y estudiantes alrededor de las tareas de cada lección, sin embargo, siguen siendo escasas estas investigaciones. Inclusive, algunos investigadores como Seckel, Breda y Font (2019) y Font, Planas y Godino (2010), sugieren criterios de idoneidad didáctica que pueden ser considerados para contribuir a la mejora de esta práctica. En México y latinoamerica muy poco se sabe de las conceptualizaciones de la pendiente previstas en el currículum planeado y en el implementado en la práctica de la enseñanza misma de la matemática, incluso del currículum que los estudiantes pueden demostrar haber aprendido. De ahí la pertinencia de que futuras investigaciones centren la atención en estas cuestiones.

\section{Referencias}

BARDIN, L. El análisis de contenido. 3. ed. Madrid: Akal, 2002. 
BARMBY, P.; HARRIES, T.; HIGGINS, S.; SUGGATE, J. The array representation and primary children's understanding and reasoning in multiplication. Educational Studies in Mathematics, Dordrecht, v. 70, n. 3, p. 217-241, 2009.

BERRY, J.; NYMAN, M. Promoting students' graphical understanding of the calculus. The Journal of Mathematical Behavior, New York, v. 22, n. 4, p. 479-495, 2003.

BIRGIN, O. Investigation of eighth-grade students' understanding of the slope of the linear function. Bolema, Rio Claro, v. 26, n. 42a, p.139-162, 2012.

BROUSSEAU, G. Epistemological obstacles, problems and didactical engineering. In: BALACHEFF, N.; COOPER, M.; SUTHERLAND, R.; WARFIELD, V. (ed.). Theory of Didactical Situations in Mathematics. New York: Kluwer Academic Publishers, 2002. p. 79-117.

BUSINSKAS, A. Conversations about connections: How secondary mathematics teachers conceptualize and contend with mathematical connections. 2008. Thesis (Doctoral in Philosophy) Faculty of Education-Simon Fraser University, Burnaby, 2008.

CAI, J.; DING, M. On mathematical understanding: perspectives of experienced Chinese mathematics teachers. Journal of Mathematics Teachers Education, Dordrecht, v. 20, n. 1, p. 1-25, 2015.

CASEY, A.; NAGLE, C. Students' use of slope conceptualizations when reasoning about the line of best fit. Educational Studies in Mathematics, Dordrecht, v. 92, n. 2, p.163-177, 2016.

CHO, P.; NAGLE, C. (2017). An analysis of students' mistakes on routine slope tasks. In: GALINDO, E. and NEWTON, J., (ed.). Proceedings of the 39th annual meeting of the North American Chapter of the International Group for the Psychology of Mathematics Education. Indianapolis: Hoosier Association of Mathematics Teacher Educators, 2017. p. 645-652.

CHOY, B.; LEE, M.; MIZZI, A. Textbook signatures: an exploratory study of the notion of gradient in Germany, Singapore and South Korea. In: BESWICK, K.; MUIR, T.; WELLS, J. (ed.) Proceedings of the $3^{\text {th }}$ Conference of the International group for the Psychology of Mathematics Education. Hobart: International Group for the Psychology of Mathematics Education, v. 2, 2015. p. 161-168.

CORDERO, F.; CEN, C.; SUÁREZ, L. Los funcionamientos y formas de las gráficas en los libros de texto: una práctica institucional en el bachillerato. Revista Latinoamericana de Investigación en Matemática Educativa, México, D. F., v. 13, n. 2, p. 187-214, 2010.

CORDERO, F.; FLORES, R. El uso de las gráficas en el discurso matemático escolar: Un estudio socioepistemológico en el nivel básico a través de los libros de texto. Revista Latinoamericana de Investigación en Matemática Educativa, México, D. F., v. 10, n. 1, p. 7-38, 207.

COURTNEY, N.; MARTÍNEZ-PLANELL, R.; MOORE-RUSSO, D. Using APOS theory as a framework for considering slope understanding. Journal of Mathematical Behavior, New York, v. $54,2019$.

DENIZ, Ö.; KABAEL, T. $8^{\text {th }}$ Grade students' construction processes of the concept of slope. Education and Science, Ankara, v. 42, n. 192, p. 139-172, 2017. Disponible en:http://egitimvebilim.ted.org.tr/index.php/EB/article/view/6996. Acceso en: 19 oct. 2019.

DOLE, S.; SHIELD, M. J. The capacity of two Australian eighth-grade textbooks for promoting proportional reasoning. Research in Mathematics Education, London, v. 10, n. 1, p. 19-35, 2008. 
DOLORES, C.; RIVERA, M. I.; GARCÍA, J. Exploring mathematical connections of pre- university students through tasks involving rates of change. International Journal of Mathematical Education in Science and Technology, London, v. 50, n. 3, p. 369-389, 2019.

DOLORES, C.; RIVERA, M. I.; MOORE-RUSSO, D. Conceptualizations of slope in Mexican intended curriculum. School Science and Mathematics, Hoboken, v. 120, n. 2, p. 104-115, 2020

FAN, L.; ZHU, Y.; MIAO, Z. Textbook research in mathematics education: development status and directions. ZDM, Hamburg, v. 45, n. 5, p. 633-646, 2013.

FLODEN, R. The measurement of opportunity to learn. In: PORTER, A.; GAMORAN, A. (ed.). Methodological advances in cross-national surveys of educational achievement. Washington: National Academy Press, 2002. p. 231-266.

FONT, V.; PLANAS, N.; GODINO, J. Modelo para el análisis didáctico en educación matemática. Infancia y aprendizaje, Madrid, v. 33, n. 1, p. 89-105, 2010.

FUENLABRADA, S.; FUENLABRADA, I. R. Geometría Analítica. 4. ed., México, D. F., Mc Graw Hill Education, 2014.

GEHRKE, N. J.; KNAPP, M. S.; SIROTNIK, K. A. In search of the school curriculum. Review of Research in Education, Washington, v. 18, n. 1, p. 51-110, 1992.

MÉXICO. SEP. Acuerdo número 444 por el que se establecen las competencias que constituyen el marco curricular común del Sistema Nacional de Bachillerato. Diario Oficial, 2008. Disponible en: http://www.sems.gob.mx/es mx/sems/acuerdo_secretarial. Acceso en: 10 sep. 2019.

GONZÁLEZ, M.; SIERRA, M. Metodología de análisis de libros de texto de matemáticas: Los puntos críticos en la enseñanza secundaria en España durante el siglo XX. Enseñanza de las Ciencias, Barcelona, v. 22, n. 3, p. 389-408, 2004.

HERBEL-EISENMANN, B. A. From intended curriculum to written curriculum: Examining the "voice" of a mathematics textbook. Journal for Research in Mathematics Education, Reston, v. 38, n. 4, p. 344-369, 2007.

HIEBERT, J.; CARPENTER, T. P. Learning and teaching with understanding. In: GROUWS, D. (ed.). Handbook of research on mathematics teaching and learning. New York: McMillan, 1992. p. 65-97.

HIEBERT, J; LEFEVRE, P. Conceptual and procedural knowledge in mathematics: an introductory analysis. In: HIEBERT, J. (ed.). Conceptual and procedural knowledge: the case of mathematics. Hillsdale: Lawrence Erlbaum Associates, 1986. p. 1-27.

HOFFMAN, W. Concept image of slope: Understanding middle school mathematics teachers' perspective through task-based interviews. 2015. Thesis (Doctoral of Philosophy in Curriculum and Instruction) - Faculty of Education, University of North Carolina, Charlotte, 2015.

HONG, D. S.; CHOI, M. K. A comparative analysis of linear functions in Korean and American standards-based secondary textbooks. International Journal of Mathematical Education in Science and Technology, London, v. 49, n. 7, p. 1025-1051, 2018.

LEHMANN, C. H. Geometría Analítica. México, D. F.: Limusa, 1980.

LINGEFJÄRD, T.; FARAHANI, D. The Elusive Slope. International Journal of Science and Mathematics Education, Kaohsiung, v.16, n. 6, p.1187-1206, 2018. 
LOBATO, J.; SIEBERT, D. Quantitative reasoning in a reconceived view of transfer. The Journal of Mathematical Behavior, New York, v. 21, n. 1, p. 87-116, 2002.

LOBATO, J.; THANHEISER, E. Developing understanding of ratio-as-measure as a foundation of slope. In: LITWILLER, B.; BRIGHT, G. (ed.). Making sense of fractions, ratios, and proportions. Reston: The National Council of Teachers of Mathematics, 2002. p. 162-175.

MANOUCHEHRI, A.; GOODMAN, T. Mathematics curriculum Reform and teachers: Understanding the connections. The Journal of Educational Research, Philadelphia, v. 92, n. 1, p. 27-41, 1998.

MÉNDEZ, A. Matemáticas III. 2. ed. México D. F.: Editorial Santillana S. A. de C. V, 2013.

MOORE-RUSSO, D.; CONNER, A.; RUGG, K. Can slope be negative in 3-space? Studying concept image of slope through collective definition construction. Educational Studies in Mathematics, Dordrecht, v. 76, n. 1, p. 3-1, 2011.

NCTM. Principles and Standards for School Mathematics. Reston: NCTM. 2000.

NAGLE, C.; CASEY, S.; MOORE-RUSSO, D. Slope and line of best fit: A transfer of knowledge case study. School Science and Mathematics, Hoboken, v. 117, n. 1-2, p. 13-26, 2017.

NAGLE, C.; MOORE-RUSSO, D. Connecting Slope, Steepness, and Angles. Mathematics Teacher, Reston, v. 107, n. 4, p. 272-279, $2013 \mathrm{a}$.

NAGLE, C.; MOORE-RUSSO, D. Slope: a network of connected components. In: MARTINEZ, M.; CASTRO, A. (ed.). Proceedings of the $3^{\text {th }}$ annual meeting of the North American Chapter of the International Group for the Psychology of Mathematics Education. Chicago: University of Illinois at Chicago, 2013b. p. 127-135.

NAGLE, C.; MOORE-RUSSO, D. The concept of slope: Comparing teachers' concept images and instructional content. Investigations in Mathematics Learning, Philadelphia, v. 6, n. 2, p. 1-18, $2013 c$.

NAGLE, C.; MOORE-RUSSO, D.; STYERS, J. L. Teachers' Interpretations of Student Statements about Slope. In: GALINDO, E.; NEWTON, J. (ed.). Proceedings of the $39^{\text {th }}$ annual meeting of the North American Chapter of the International Group for the Psychology of Mathematics Education. Indianapolis: Hoosier Association of Mathematics Teacher Educators, 2017. p. 589-596.

NAGLE, C.; MOORE-RUSSO, D.; VIGLIETTI, J.; MARTIN, K. Calculus students' and instructors' conceptualizations of slope: a comparison across academic levels. International Journal of Science and Mathematics Education Dordrecht, v. 11, n. 6, p. 1491-1515, 2013.

NOSS, R.; HEALY, L.; HOYLES, C. The construction of mathematical meanings: Connecting the visual with the symbolic. Educational Studies in Mathematics, Dordrecht, v. 33, n. 2, p. 203-233, 1997.

OHLSSON, S. Mathematical meaning and applicational meaning in the semantics of fractions and related concepts. In: HIERBERT, J.; BEHR, M. (ed.). Number Concepts and Operations in the Middle Grades. Reston: NCTM, 1988. p. 53-92.

PLANINIC, M.; MILIN-SIPUS, Z.; KATIC, H.; SUSAC, A.; IVANJEK, L. Comparison of student understanding of line graph slope in physics and mathematics. International Journal of Science and Mathematics Education, Dordrecht, v.10, n. 6, p. 1393-1414, 2012. 
REMILLARD, J. Examining key concepts in research on teachers' use of mathematics curricula. Review of Educational Research, Washington, v. 75, n. 2, p. 211-246, 2005.

REMILLARD, J.; HECK, D. Conceptualizing the curriculum enactment process in mathematics education. ZDM, Hamburg, v. 46, n. 5, p. 705-718, 2014.

RITTLE-JOHNSON, B.; SCHNEIDER, M. Developing conceptual and procedural knowledge of mathematics. In: COHEN, R.; DOWKER A. (ed.). Oxford handbook of numerical cognition. Oxford: Oxford University Press, 2015. p. 1118-1134.

RIVERA, M. I; SALGADO, G.; DOLORES, C. Explorando las conceptualizaciones de la pendiente en estudiantes universitarios. Bolema, Rio Claro, vol. 33, n.65, pp.1027-1046, 2019.

SALAZAR, P. Matemáticas 3. México. D. F.: Compañía Editorial Nueva Imagen, 2018.

SALGADO, G; RIVERA, M. I; DOLORES, C. Conceptualizaciones de pendiente: contenido que enseñan los profesores de bachillerato. UNIÓN, Revista Iberoamericana de Educación Matemática, São Paulo, v. 15, n. 57, p. 41-56, 2019.

SCHMIDT, W.; MCKNIGHT, C.; VALVERDE, G.; HSINGCHI, W.; WILEY, D.; COGAN, L.; WOLFE, R. Why schools matter: A cross-national comparison of curriculum and learning. San Francisco: Jossey-Bass Education, 2001.

SHULMAN, L. S. Those who understand: Knowledge growth in teaching. Educational Researcher, Washington, v.15, n. 2, p. 4-14, 1986.

SECKEL, M.; BREDA, A.; FONT, V. Los criterios de idoneidad didáctica en la formación de profesores. En: GARCÍA, D. J; PÉREZ, I. E.; (ed.). Acta Latinoamericana de Matemática Educativa. México, D. F.: Comité Latinoamericano de Matemática Educativa, 2019. p. 440-447.

SIERPINSKA, A. Obstacles épistémologiques relatifs à la notion de limite. Recherches en didactique des mathématiques, París, v. 6, n. 1, p. 5-67, 1985.

STACEY, K.; VINCENT, J. Modes of reasoning in explanations in Australian eighth-grade mathematics textbooks. Educational Studies in Mathematics, Dordrecht, v. 72, n. 3, p. 271-288, 2009.

STANTON, M.; MOORE-RUSSO, D. Conceptualizations of Slope: A Review of State Standards. School Science and Mathematics, Hoboken, v. 112, n. 5, p. 270-277, 2012.

STEWART, J. Cálculo de una variable, Trascendentes tempranas. México, D. F.: Cengage Learning, 2012.

STUMP, S. High school precalculus students' understanding of slope as measure. School Science and Mathematics, Hoboken, v. 101, n. 2, p. 81-89, 2001.

STUMP, S. Secondary mathematics teachers' knowledge of slope. Mathematics Education Research Journal, Sydney, v. 11, n. 2, p. 124-144, 1999.

TALL, D.; VINNER, S. Concept image and concept definition in mathematics with particular reference to limits and continuity. Educational Studies in Mathematics, Dordrecht, v. 12, n. 2, p. $151-169,1981$.

TEUSCHER, D.; REYS, R. Slope, rate of change, and Steepness: Do students understand the concepts? Mathematics Teacher, Reston, v. 103, n. 7, p. 519-524, 2010. 
TEUSCHER, D.; REYS, R. Rate of Change: AP Calculus Students ${ }^{e e}$ Understandings and Misconceptions after Completing Different Curricular Paths. School Science and Mathematics, Hoboken, v. 112, n. 6, p. 359-376, 2012.

THOMPSON, P. W. A theoretical model of quantity-based reasoning in arithmetic and algebra Center for Research in Mathematics \& Science Education: San Diego State University.Disponible en: http://pat-thompson.net/PDFversions/1990TheoryQuant.pdf. Acceso en: 8 sep 2019.

THOMPSON, P.; CARLSON, M. Variation, Covariation, and Functions: Foundational Ways of Thinking Mathematically. In: CAI, J. (ed.). Compendium for Research in Mathematics Education. Reston: NCTM, 2017. p. 421-456.

THOMPSON, P. W.; THOMPSON, A. G. Images of rate. Center for Research in Mathematics and Science Education and Department of Mathematical Sciences. San Diego State University, 15 de abril de 1992. Disponible en:

http://citeseerx.ist.psu.edu/viewdoc/download?doi=10.1.1.644.9932\&rep=rep1\&type=pdf. Acceso en: 13 oct 2019.

VALVERDE, G.; BIANCHI, L.; WOLFE, R.; SCHMIDT, W.; HOUANG, R. According to the book: Using TIMSS to investigate the translation of policy into practice through the world of textbooks. Dordrecht: Kluwer Academic Publishers, 2002.

VAN STEENBRUGGE, H.; VALCKE, M.; DESOETE, A. Teachers' views of mathematics textbook series in Flanders: Does it (not) matter which mathematics textbook series schools choose? Journal of Curriculum Studies, London, v. 45, n. 3, p. 322-353, 2013.

VERGNAUD, G. Multiplicative structures. In: LESH, R.; LANDAU, M. (ed.). Acquisition of mathematics concepts and processes. New York: Academic Press, 1983. p. 127-174.

WEBER, E.; TALLMAN, M.; MIDDLETON, J. Developing elementary teachers' knowledge about functions and rate of change through modeling. Mathematical Thinking and Learning, London, v. 17, n. 1, p. 1-33, 2015. 